Supporting Information

\title{
Enhanced Molecular Packing of a Conjugated Polymer with High Organic Thermoelectric Power Factor
}

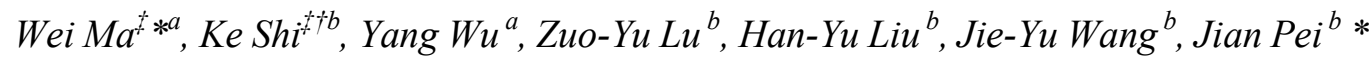

${ }^{\text {a }}$ State Key Laboratory for Mechanical Behavior of Materials, Xi'an Jiaotong University, Xi'an 710049, China

${ }^{b}$ Beijing National Laboratory for Molecular Sciences, Key Laboratory of Bioorganic Chemistry and Molecular Engineering of Ministry of Education, Key Laboratory of Polymer Chemistry and Physics of the Ministry of Education, Center for Soft Matter Science and Engineering, College of Chemistry and Molecular Engineering, Peking University, Beijing 100871, China

\section{Corresponding Author}

E-mail: $\underline{\text { msewma@xjtu.edu.cn }}$

E-mail: jianpei@pku.edu.cn 
(a)

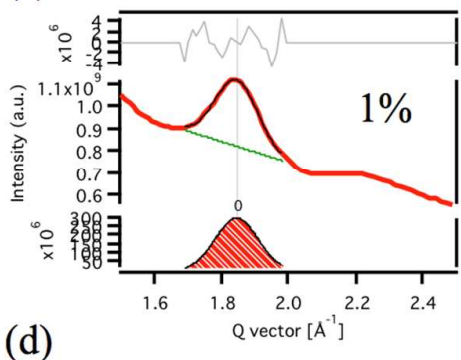

(d)

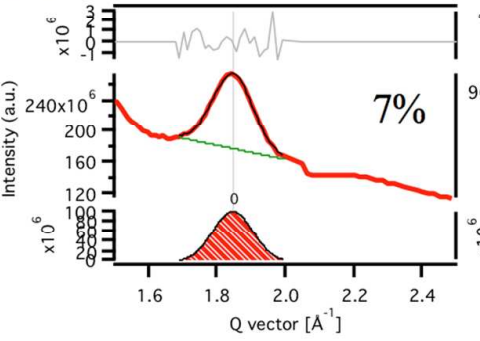

(b)

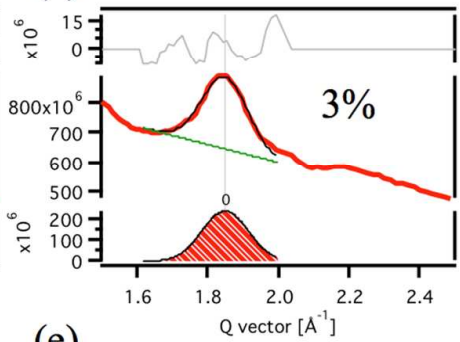

(e)

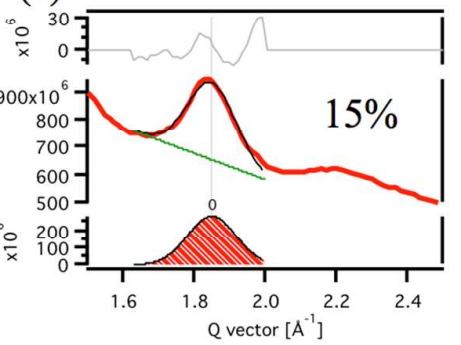

(c)

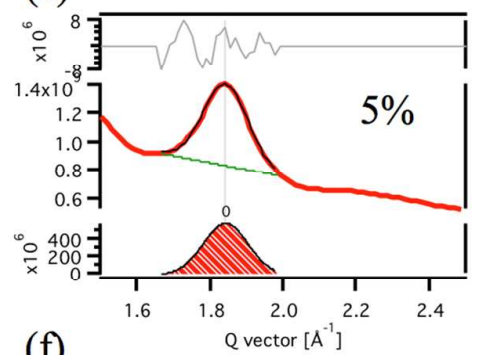

(f)

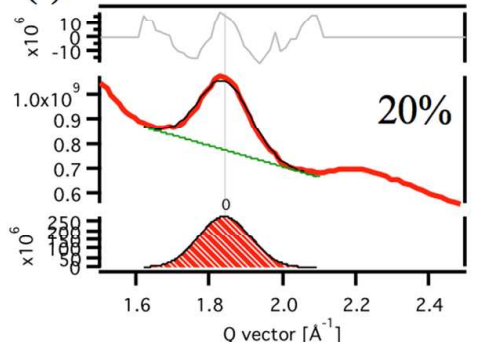

Figure S1. The fitted (010) peaks for the FBDPPV blended with dopant at different amount.
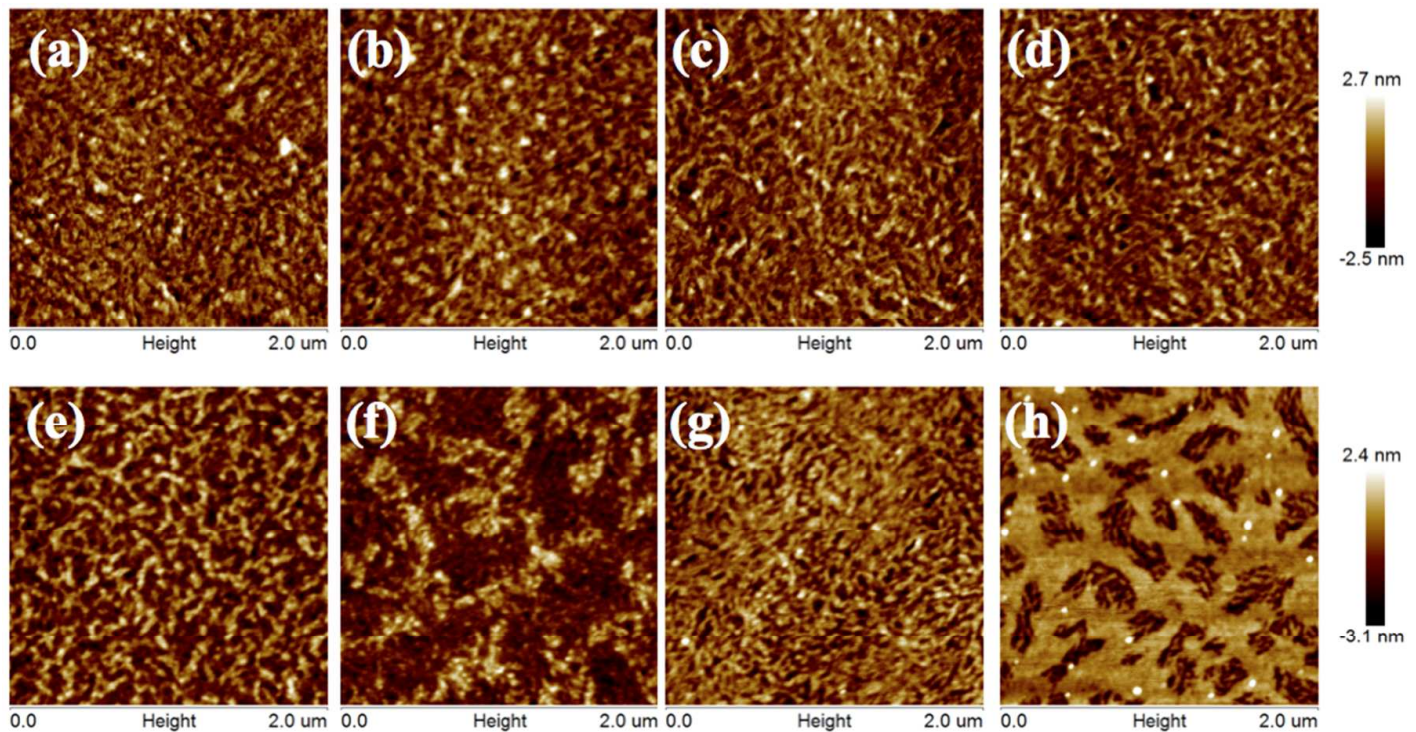

Figure S2: AFM high images of (a) pure FBDPPV, (b-h) FBDPPV doped with 1 wt \%,

3 wt $\%, 5$ wt $\%, 7$ wt \%, 15 wt \%, 20 wt $\%$ and 50 wt $\%$ N-DMBI. 


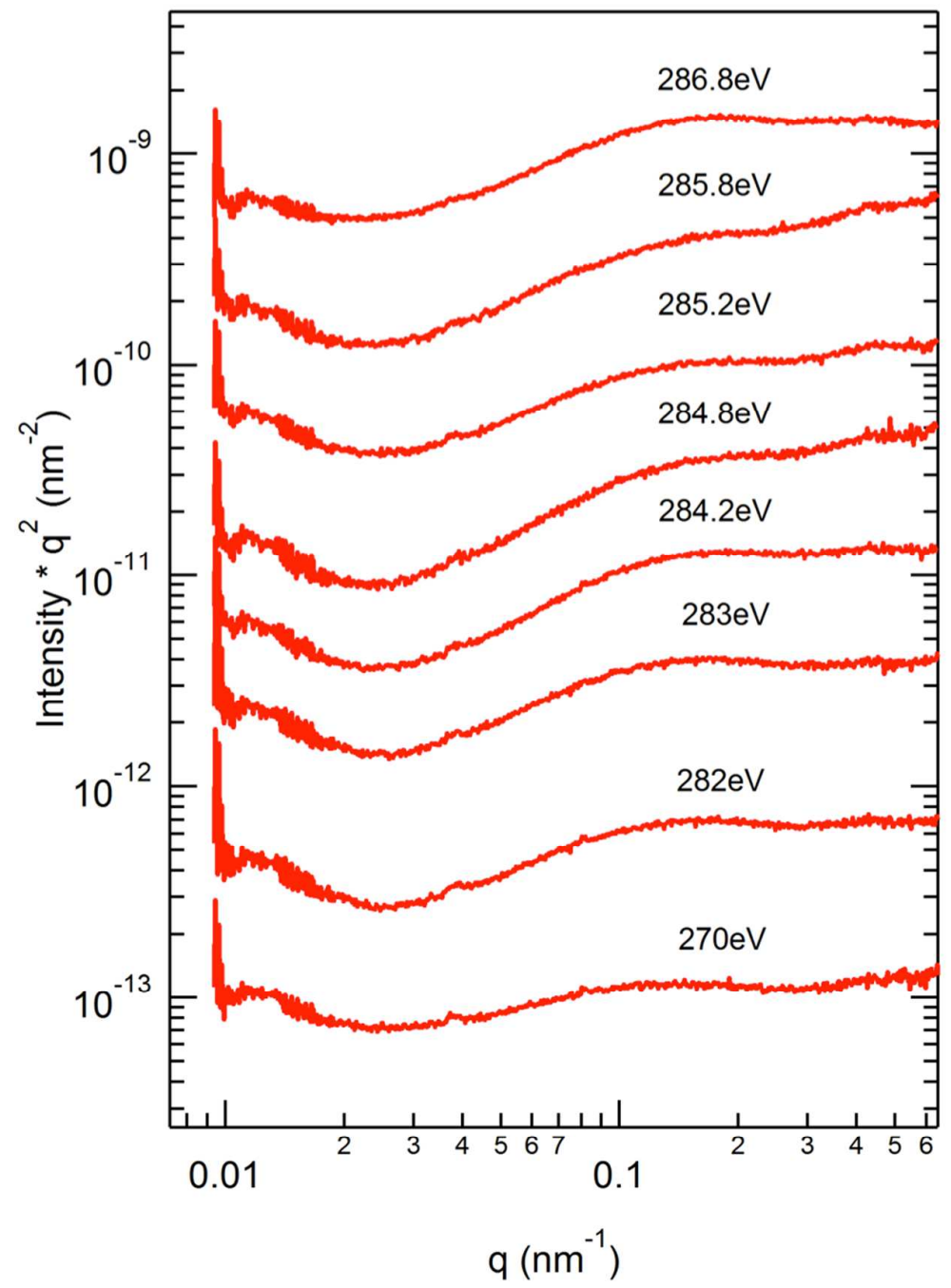

Figure S3: R-SoXS scattering at 270, 282, 283, 284.2, 284.8, 285.2, 285.8 and 286.8 $\mathrm{eV}$ of the $5 \%$ dopant. 


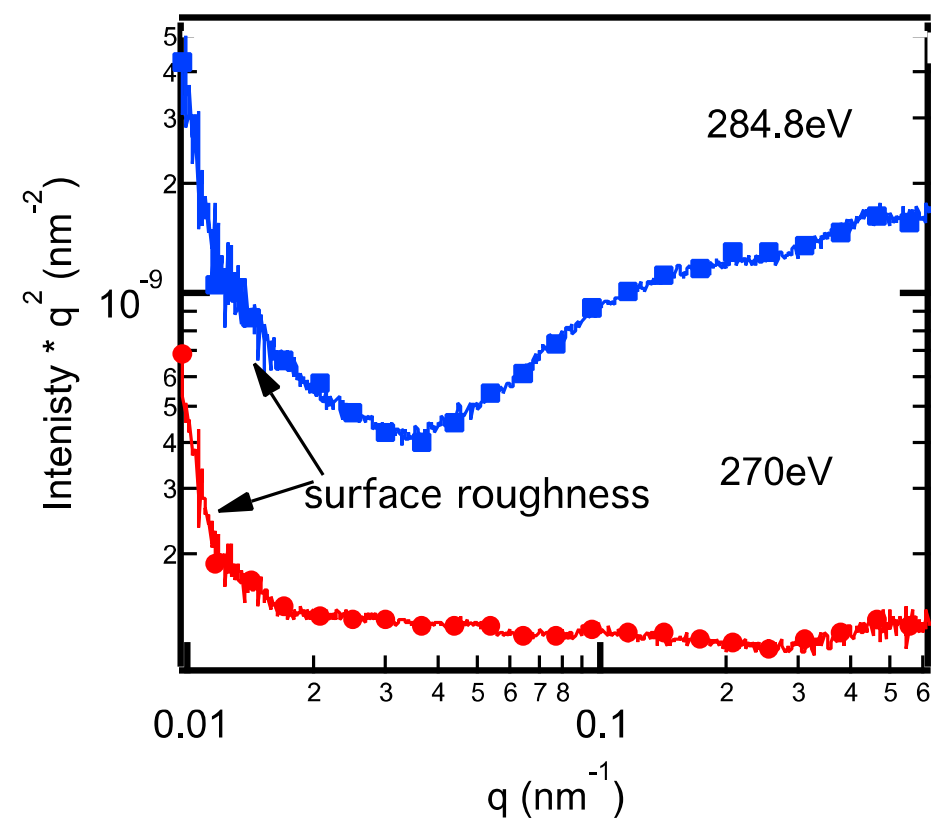

Figure S4: R-SoXS scattering at $270 \mathrm{eV}$ (vacuum:materials contrast is high) and $284.8 \mathrm{eV}$ (materials:materials contrast is high) of $15 \%$ dopant.

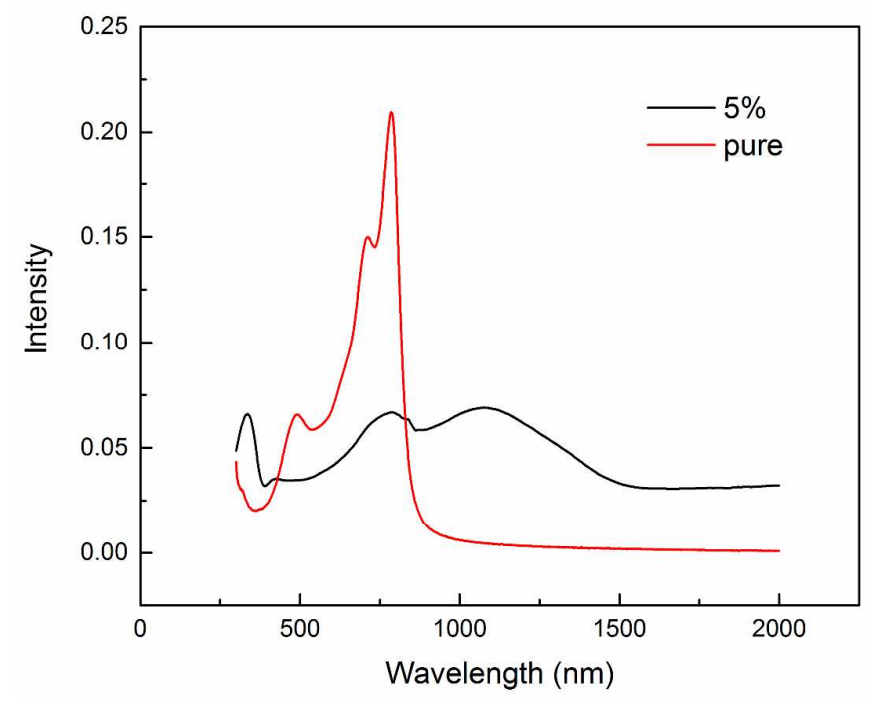

Figure S5: The UV-Vis absorption spectra of doped (5\%) and un-doped FBDPPV thin film. 


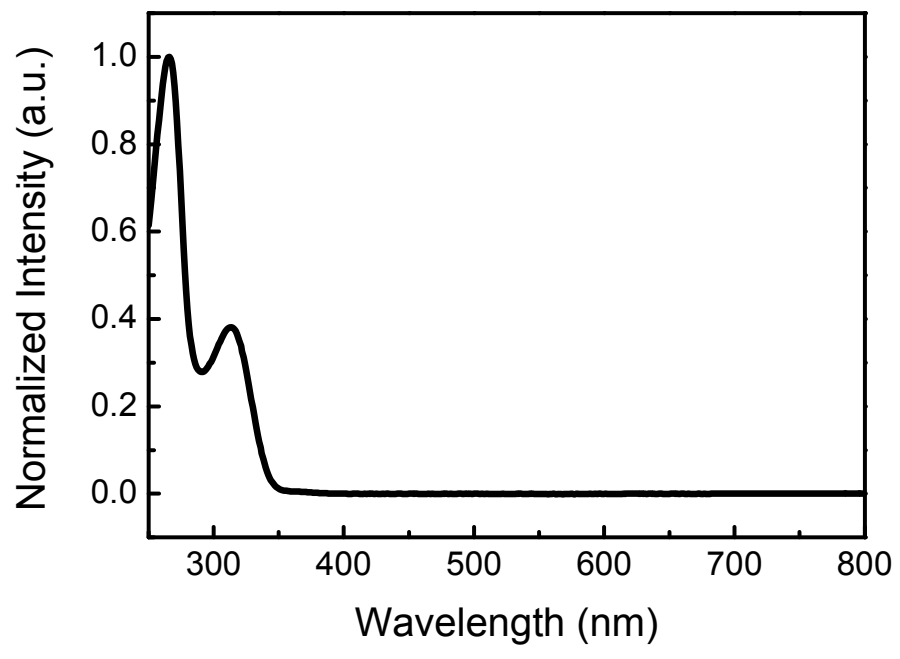

Figure S6. Absorption spectra of dopant N-DMBI in $\mathrm{CH}_{2} \mathrm{Cl}_{2}\left(10^{-4} \mathrm{M}\right)$.

Molecular ratio (DMBI molecule being 2:1 - 3:1)

In the previous mechanistic study on the solution-phase n-doping of N-DMBI by Prof.

Bao, they proposed a mechanism of a bimolecular hydride transfer from dopant to PCBM yielding the $\mathrm{DMBI}^{+}, \mathrm{PCBM}^{-}$, and side product $\mathrm{PCBMH}_{\mathrm{x}}$. The doping reaction is as below:

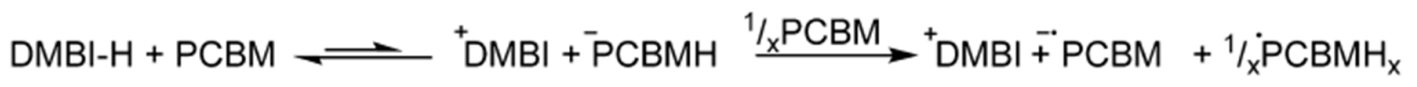

As a consequence, the doping reaction may attain the best level when $\mathrm{x}=1$, that is, when the molar concentration of PCBM: DMBI-H is 2:1. In our system, the molecular weight of FBDPPV repeat unit is $1630 \mathrm{~g} / \mathrm{mol}$, and the molecular weight of N-DMBI is $267 \mathrm{~g} / \mathrm{mol}$. Therefore, the $5-7 \mathrm{wt} \%$ weight fraction corresponds to the ratio between the number of the FBDPPV repeat unit and the number of the N- 